\title{
A data analysis library for gravitational wave detection
}

\author{
A. Lassus ${ }^{1}$, R. van Haasteren ${ }^{2}$, C. M. F. Mingarelli ${ }^{3}$, K. J. Lee ${ }^{4}$ and \\ A. Vecchio $^{3}$ \\ ${ }^{1}$ LPC2E, CNRS, Université d'Orléans \\ email: antoine.lassus@cnrs-orléans.fr \\ ${ }^{2}$ Max-Planck-Institut für Gravitationsphysik (Albert-Einstein-Institut), D-30167 Hannover, \\ Germany \\ email: vhaasteren@gmail.com \\ ${ }^{3}$ School of Physics and Astronomy, University of Birmingham, Edgbaston, \\ Birmingham B15 2TT, UK \\ email: chiara@star.sr.bham.ac.uk, av@star.sr.bham.ac.uk \\ ${ }^{4}$ Max-Planck-Institut für Radioastronomie, Auf dem Hügel 69, D-53121 Bonn, Germany \\ email: kjlee@mpifr-bonn.mpg.de
}

\begin{abstract}
One of the main goals of Pulsar Timing Arrays (PTAs) is the direct detection of gravitational waves (GWs). A first detection will be a major leap for astronomy and substantial effort is currently going into timing as many pulsars as possible, with the highest possible accuracy. As part of the individual PTA projects, several groups are developing data analysis methods for the final stage of a gravitational-waves search pipeline: the analysis of the timing residuals. Here we report the progress of on-going work to develop, within a Bayesian framework, a comprehensive and user friendly analysis library to search for gravitational waves in PTA data.
\end{abstract}

Keywords. pulsars: general, gravitational waves, methods: data analysis

\section{Introduction}

High precision timing of millisecond pulsars holds great promise for the detection of gravitational waves (GWs) in the $10^{-9}-10^{-7} \mathrm{~Hz}$ frequency range, which includes the inspiral regime of Super Massive Black Holes Binaries (SMBHBs). GWs affect the time of arrival of the radio pulses coming from pulsars in the PTA (see Estabrook \& Wahlquist 1975, Sazhin 1978, Detweiler 1979). The GWs perturb the propagation of the observed radio emission in such a way that the arrival time of the pulses only depends on the metric perturbation at the pulsar at the moment of emission (the "pulsar term"), and the metric perturbation at the Earth at the point of reception (the "Earth term"). Due to the continuous monitoring of many pulsars, the Earth term is correlated between every pulsar pair in a unique way described by General Relativity. Hellings \& Downs (1983) have shown what form these correlations have for an isotropic stochastic background of GWs, such as the one generated by an unresolved cosmic population of SMBHBs. The spectral density of this signal follows a power law (see Maggiore 2000, Phinney 2001) and is defined in terms of its characteristic strain:

$$
h_{c}=A\left(\frac{f}{y r^{-1}}\right)^{\alpha},
$$

where $A$ is the signal amplitude, $f$ its frequency and $\alpha$ the spectral index. 
Besides the stochastic GWB, there are other possible signals in the observations which one should consider: GWs from individually resolvable sources (Sesana \& Vecchio 2010, Mingarelli et al. 2012, Ellis et al. 2012), cosmic strings (Sanidas et al. 2012), GW bursts (Pitkin 2012), imperfections in terrestrial time standards (Hobbs et al. 2012), and dispersion measure variations. New methods to look for these signals are continuously being developed, but to date there is no easy to use open data analysis pipeline specifically designed to combine all these in a single pipeline. The purpose of this work is to provide such a versatile and modular pipeline for the pulsar timing community.

\section{Example}

An example of general pulsar timing analysis is the correct treatment of time- correlated stochastic signals, like red timing noise, in pulsar timing data. In solving for the timing model parameters, a lot of the timing noise is absorbed in the fitting procedure. Several consistent methods now exist to treat red timing noise: among them the Cholesky method of Coles et al. (2011), and the Bayesian method of Haasteren et al. $(2009,2012)$. In the panel above the reconstruction of some simulated low frequency

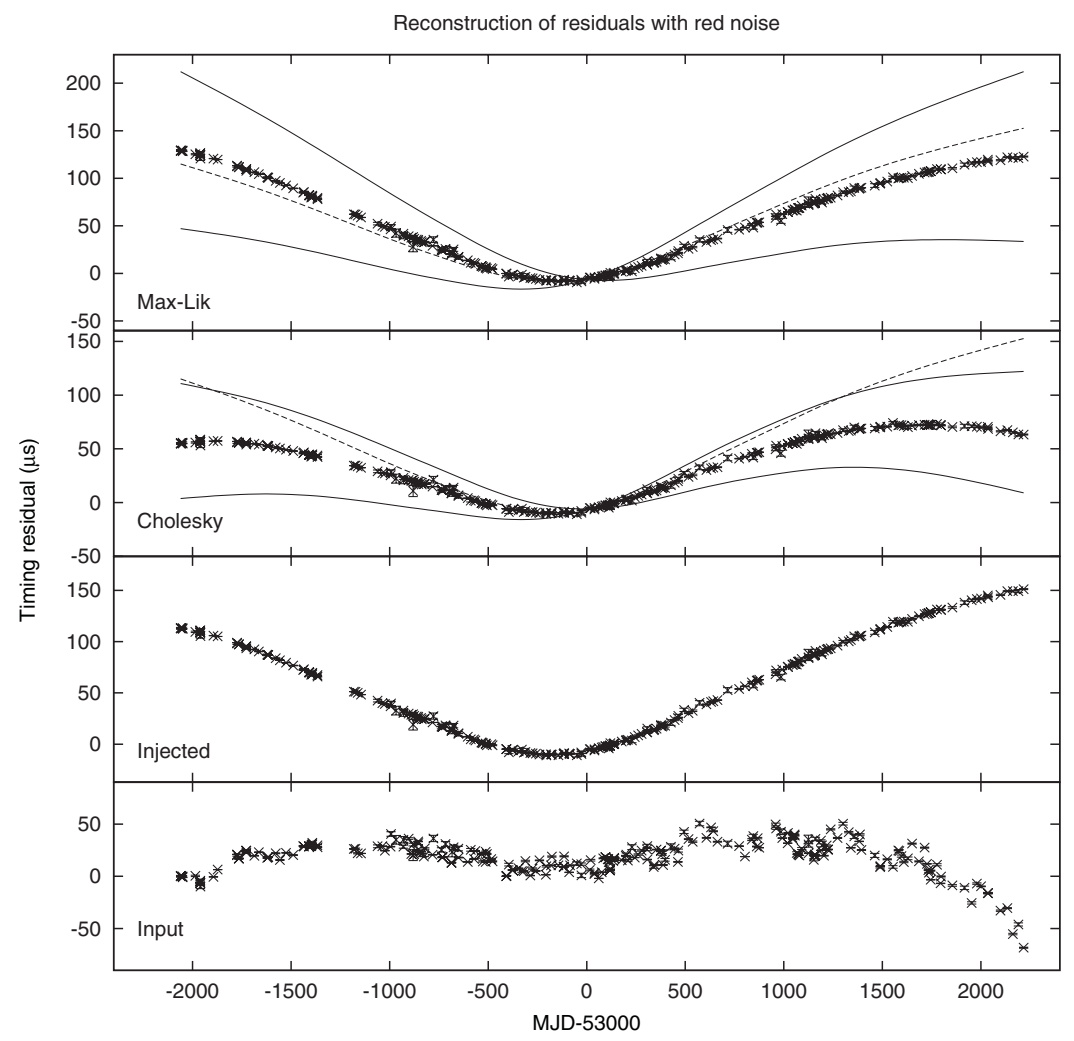

Figure 1. Different reconstructions of injected low frequency noise. The bottom panel is the input of the residuals without any pre-processing (input). The second lowest shows the injected form of the residuals containing the low frequency noise (injected). The top panel shows the reconstruction with the Maximum likelihood method (Max-Lik), the second highest one shows a reconstruction using the Cholesky method (Cholesky). For the reconstructions, we have plotted the $1 \sigma$ intervals for the pulse frequency; the lowest frequency timing model parameter. For the Maximum likelihood estimator, the injected signal seems to lie more within this interval. 
timing noise is presented for the Cholesky method, and the maximum likelihood (ML) method of van Haasteren (2012). Correct treatment of the timing noise, especially for the low-frequency behaviour, is essential for gravitational-wave detection. Both methods will be easily accessible in the new data analysis library.

\section{Structure of the data analysis process}

The goal of our software library, developed in python and C, is to be as user-friendly as possible. At the moment, no uniform pipeline or software library is publicly available for this kind of analysis. The purpose of this work is to be able to combine different models and different algorithms in the simplest way.

The key element of such a pipeline is the likelihood evaluator, which implements the likelihood function. The likelihood evaluator will be implemented as part of a modular library, where each of the modules can be easily replaced with an alternative. Different samplers, and signal models can therefore be easily combined without having to make changes in the library. Standard modules will be provided for the treatment of typical analysis cases, including GW(B) detection, red noise estimation, and dispersion measure variation correction. Future models will include GWs from single sources, bursts and cosmic strings.

\section{Conclusion}

We present a new library, still under construction, designed to become a universal tool for the analysis of pulsar timing data. The library has a special focus on pulsar timing array science and GW detection, but will also contain useful methods for pulsar timing in general. Typical problems that this library is especially well-suited for are pulsar timing data analysis in the presence of strong low-frequency noise and GW detection. Independent library modules can be easily combined and replaced, which allows different types of analysis to be done without having to change any code. Such modules include, but are not limited to, the modules implementing Markov Chain Monte Carlo samplers, and modules describing the GW signal model in the likelihood function. We will provide standard modules for many common data analysis problems, so that a typical analysis can be done "out of the box".

\section{References}

W. Coles, et al. MNRAS 418, 561 .

S. Detweiler, 1979, ApJ, 234, 1100.

J. Ellis, et al., ApJ 753, 96E

F. B. Estabrook \& H. D. Wahlquist, 1975, Gen. Relativ. Gravit., 6, 439.

R. W. Hellings \& G. S. Downs, 1983, ApJ, 265, L39.

G. Hobbs, et al., 2012, arXiv:astro-ph/1208.3560

M. Maggiore, 2000, Phys. Rep., 331, 6

C. M. F. Mingarelli, et al., 2012, Phys. Rev. Lett 109, 081104

E. S. Phinney, 2001, arXiv:astro-ph/0108028

M. Pitkin , 2012, arXiv:astro-ph/1201.3573

S. A. Sanidas, et al., 2012, Phys. Rev. D, 85, 122003.

M. V. Sazhin, 1978, Sov. Astron., 22, 36.

A. Sesana \& A. Vecchio, 2010, Phys. Rev. D, 81, 104008.

A. Sesana, A. Vecchio \& C. N. Colacino,, 2008, MNRAS, 390, 192.

R. van Haasteren, et al., 2009, MNRAS 395, 1005

R. van Haasteren, 2012, arXiv:1210.0584 